\title{
Quality improvement cycles that reduced waiting times at Tshwane District Hospital Emergency Department
}

\author{
${ }^{a}$ Rauf W, BSc, MBBS, DipPEC(SA), MMed(FamMed)UP \\ a Blitz JJ, BSc, MBBCh(Wits), MPraxMed(MED) \\ a Geyser MM, BSc, DipPEC(SA), BSc(Pharm)Hons, MPraxMed, FCEM(SA), MSc(Clin Epi) \\ ${ }^{b}$ Rauf A, MBBS, FRCS(Ire), HDip(SA), FRCS(Glasg) \\ a Department of Orthopaedics, University of Pretoria \\ ${ }^{\mathrm{b}}$ Department of Orthopaedics, University of Pretoria
}

Corespondence to: Dr W Rauf, e-mail: nisa.rauf@up.ac.za

Abstract

Background: Tshwane District Hospital (TDH) is a level-one hospital, delivering services in the centre of Pretoria since February 2006. It is unique in location, being only 100 meters away from the tertiary hospital, Pretoria Academic Hospital (PAH). In South Africa, public sector emergency units are under enormous pressure with large patient numbers, understaffing and poor resources. TDH Emergency Department (ED) is a typical example. An average of 3900 patients per month visited this ED in 2006. Recurrent complaints and dissatisfaction shown by the patients about prolonged waiting times before consulting the medical practitioners (MPs) in the ED were one of the initial challenges faced by the newly established hospital. It was decided to undertake quality improvement (QI) cycles to analyse and improve the situation, using waiting time as a measure of improvement.

Methods: A QI team was chosen to conduct two QI cycles. The allocated time for QI cycle 1 was from May to August 2006 and for QI cycle 2 from September to December 2006. A total of 150 waiting times of stable and unstable patients were evaluated. Fifty waiting times were recorded over a span of 24 hours for each data collection in May, September and December 2006. Waiting time was defined as the time from arrival of the patient in the unit until the start of the consultation by the MP. Surveys were done in May and September to analyse the problems causing prolonged waiting times. The implemented change included instituting a functional triage system, improvement of the process of up- and down-referrals to and from the tertiary hospital, easy access to stock, reorganisation of doctors' duty roster, reorganisation of the academic programme, announcement on waiting time to patients, nurses carrying out minor procedures and availability of reference books.

Results: The median waiting times for stable patients were as follows: May 2006: 545 minutes (range 200 to 1260 ), September 2006: 230 minutes (range 15 to 480) and December 2006: 89 minutes (range 15 to 230). There was a significant difference among these waiting times for May, September and December 2006 ( $p<0.001$; Kruskal-Wallis $\mathrm{H}$ test). The median waiting times for unstable patients were as follows: May 2006: zero minutes (range 0 to 30), September 2006: zero minutes (range 0 to 3) and December 2006: 0.5 minutes (range 0 to 2). There was no difference among the waiting times for unstable patients for May, September and December 2006 ( $p=0.90$; Kruskal-Wallis $\mathrm{H}$ test).

Conclusion: This QI exercise identified problems causing prolonged waiting time for stable patients at TDH ED. It rectified most of the identified problems. However, goals regarding registration and laboratory delays could not be successfully achieved. This study showed the significance of QI cycles in improving waiting times for stable patients at TDH ED without any additional financial or human resources. This was done without compromising the time taken to see unstable patients. 
Introduction

TDH is a level-one hospital, delivering services in the centre of Pretoria since February 2006. It is unique in location, being only 100 meters away from the tertiary hospital, PAH. In addition, it is a teaching hospital affiliated with the University of Pretoria.

In South Africa, public sector emergency units are under enormous pressure with large patient numbers, understaffing and poor resources. ${ }^{1}$ TDH ED is a typical example. An average of 3900 patients per month visited this ED in 2006. ED crowding and diminishing capacity are growing concerns all over the world. ${ }^{2}$ No one likes to wait. Yet, patients have to wait in most hospitals. Waiting time varies depending on the service capacity of the particular hospital. Waiting time is also called 'customer sacrifice'. Customers must sacrifice their time and other opportunities in order to obtain desired health services. ${ }^{3}$ International literature shows mean waiting times of 38 minutes in Chicago ${ }^{4}$ and 56 minutes in California. ${ }^{5}$ West Indies ED reported median waiting times of 178 minutes. ${ }^{6}$ According to a survey in the United Kingdom $66 \%$ of patients wished to see a doctor within two hours of arrival. ${ }^{7}$ South African targets for waiting time are based on the severity of the condition. Very sick unstable (priority one) patients should be seen immediately on arrival and for stable patients a maximum waiting time of 120 minutes is suggested in the ED. ${ }^{8}$

Recurrent complaints by the patients about prolonged waiting times in the TDH ED were one of the major challenges faced by the hospital. Literature shows that prolonged waiting time in the ED causes patient dissatisfaction and complaints. ${ }^{9}$ Overcrowding of patients creates anxiety and job dissatisfaction amongst healthcare providers, as they have to deal with many patients in a limited time. ${ }^{2}$ This situation was encountered at TDH. Therefore, prolonged waiting time was identified as the initial problem that needed to be addressed.

Waiting time is considered a measurable parameter for checking the efficiency of the ED. ${ }^{10}$ In the 2006 TDH ED study, two QI cycles were undertaken, using waiting time as a measure to assess the improvement. Ql in medical practices is a method for continuously finding better ways to provide better care and service. ${ }^{11}$ The $\mathrm{QI}$ cycle is a recognised tool for analysing and improving the efficiency and quality of healthcare services. ${ }^{12} \mathrm{Ql}$ is a team effort, requiring knowledge, skills, experience and perspective of each team member. ${ }^{11}$ This method of improvement was undertaken as it involves the team and thus should be more acceptable and sustainable. In the Ql cycle a problem is chosen and a team who understands the process is identified to analyse the situation. A measurable parameter is decided on. The QI team agrees on criteria to set the target standards and plans and implements the changes. The team measures the effects of changes after a set time. QI cycles are like a spiral that continues until the desired standards are achieved. In each cycle, an unresolved previous problem or some new issues are addressed. ${ }^{11}$

\section{Methods}

In this study two Ql cycles were conducted. The allocated time for QI cycle 1 was from May to August 2006 and for QI cycle 2 from September to December 2006. Implementation of changes continued during and after the end of the two cycles. Figure 1 summarises the stepwise processes that were undertaken in the Ql cycles.

\section{Ql team}

A QI team was selected in order to conduct the $\mathrm{QI}$ cycle. The team included the head of the Department of Family Medicine, the hospital superintendent, the consultant in charge of the ED, the sister in charge
Figure 1: Steps for Ql cycles

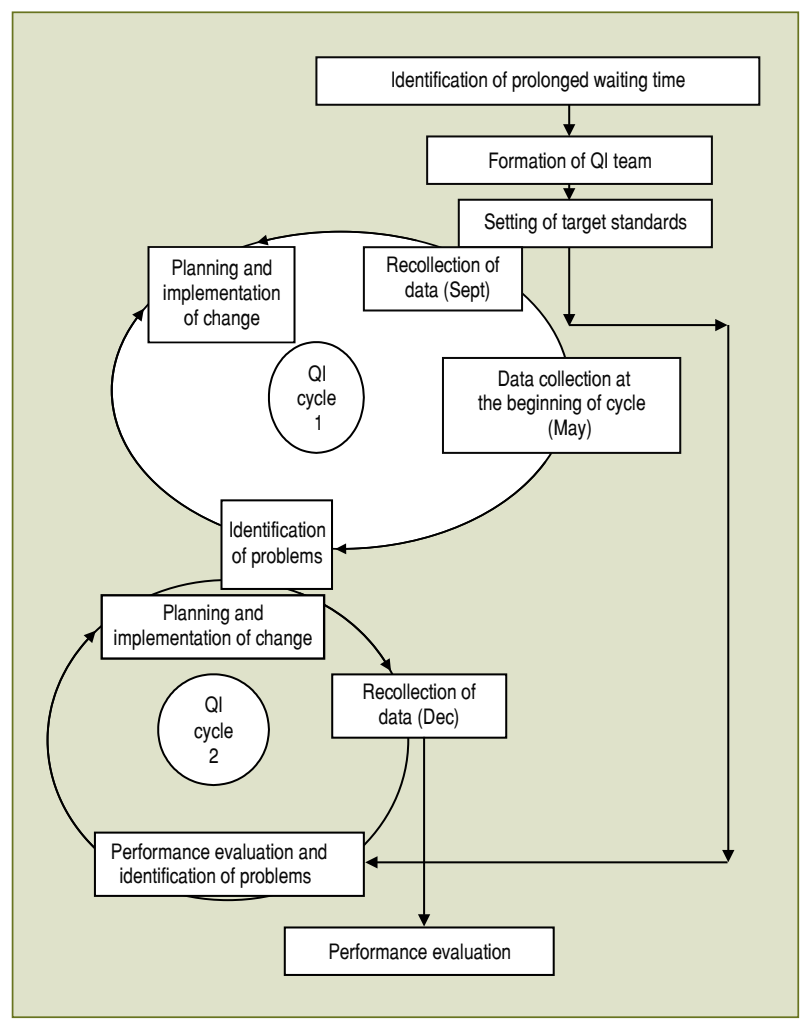

and the researcher. The QI team held weekly meetings for continuous and effective Ql cycles.

\section{Measurable variable}

Waiting time was identified as a measurable variable by the QI team. Waiting time was defined as the time from arrival of the patient in the unit until the start of the consultation by the MP. ${ }^{5}$

\section{Sampling}

Data were collected thrice during the $\mathrm{Ql}$ cycles. The first data collection was performed in May 2006 at the beginning of Ql cycle 1 to record baseline waiting times before interventions. The second and third data collections took place at the end of QI cycles 1 and 2 in September and December 2006 respectively, to evaluate the effect of interventions. Each time data were collected by convenience sampling over the span of 24 hours by the same MP who was on duty for 24 hours. No other healthcare workers in the ED were aware of the sampling. Other sampling methods were not used as the MP could not select patients to keep the process secret. Data collection was continued until a total of 50 waiting times were obtained for each shift.

The routine process was followed during sampling. The nursing sister at Reception noted the time of arrival of the patients in a register. Patients were given a sticker with a number representing their turn in the waiting queue. This number was written on the patient's file. Files were arranged according to the time of arrival. MPs had to sign and record the time on the same register at Reception before starting the consultation with the patient, as a routine process. However, MPs were not consistent in recording the time. Thus, large quantities of randomised data could not be collected. However, one MP who was motivated to do $\mathrm{Q}$ cycles strictly recorded the time. Waiting time was calculated as the difference between the time of arrival and the start of the consultation. The sister at Reception and the MP collecting samples used the clock at Reception to record the time on the register. The MP ensured optimal functioning of the clock on the sampling day. 
Patients were grouped as stable or unstable as judged by the MPs on duty in the unit, using Advanced Trauma Life Support (ATLS) criteria for priority one $(\mathrm{P} 1)$ to priority three $(\mathrm{P} 3){ }^{13} \mathrm{P} 1$ and $\mathrm{P} 2$ patients were considered unstable and $\mathrm{P} 3$ patients stable. Stable patients were attended to according to their waiting numbers. In contrast, unstable patients were taken out of the queue and attended to as soon as possible. As a routine, unstable patients were attended to immediately by the team of two MPs and available nurses. All unstable patients who presented during the sampling day were included in the data.

\section{Data analysis}

Data were analysed with Stata 8.1 software (Intercooled for Windows; STATA Corp, College Station, Tex; 2003). The Kruskall-Wallis $\mathrm{H}$ test was used as the data had a skewed distribution. A preset $P$ value of 0.05 was identified, below which statistical significance was to be established.

\section{Standards}

The following standards were set according to available evidence in the literature and taking into account the circumstances of TDH:

1. Stable patients should see the doctor within two hours of arrival. ${ }^{7,8}$

2. Unstable patients must be attended to in less than one minute (immediately). ${ }^{8}$

\section{Identification of problems and planning}

The problems causing prolonged waiting times were identified during QI cycle 1. Information was discussed amongst the team members. The following plan of action was formulated and implemented, according to the best available evidence. In QI cycle 2 the same problems were addressed and actions were reinforced.

\section{a. Triage system}

The process of sorting patients according to the severity of illness is called triage. ${ }^{1}$ The importance of triage was identified. ${ }^{14}$ The need for an effective triage system was suggested in order to maximise efficiency of the use of resources and to minimise risk to the patients. ${ }^{1}$ It was decided that patients must be triaged on arrival and streamed according to the severity of illness by a trained professional nurse or MP. ${ }^{10,11}$ Such a practitioner was identified for each shift. The severely ill patients were separated from those who were less ill and moved to a different area for care.

\section{b. Improvement in referral system}

Many patients were down-referred wrongly by $\mathrm{PAH}$. Some of them were too sick to be managed at a level-one hospital, ultimately needing tertiary care. Other patients were not resident in the service areas of TDH so could have been down-referred to other facilities. Up-referrals were difficult due to a lack of knowledge about the limitation of services available at a level-one hospital. It was decided that communication should be improved and each problem with up- or down-referral was discussed with the consultant of the relevant department at PAH.

\section{c. Standardised management textbooks}

Standardised management textbooks were made available at the doctors' desk for reference during duty hours to decrease time spent on phoning for advice from seniors and relevant registrars at PAH.

\section{d. Easy availability of stock}

The QI team observed that the stock was not stored at the right places, causing delays in finding stock for procedures. Easy availability of the stock was promoted by cooperation of the nursing staff. Unexpected checking of trolleys at various times was practised to monitor the standard of availability and functioning of equipment and stock.

e. Reorganising the duty roster

Literature shows the significance of a higher number of medical and nursing practitioners at peak hours when greater numbers of patients are expected. ${ }^{15}$ The duty roster of the doctors was reorganised to ensure the maximum number of doctors at busy times. The QI team determined the busy times by observing the patient influx in the ED over the previous months. The register at Reception and discussions with the doctors and nurses were helpful for reorganising the duty roster.

It was postulated that recurrent early morning accidents on various days of the week caused patient crowding in the ED around 07:00 to 10:00. The time for change of shift for doctors was 08:00 and for nurses 07:00. The doctors working the previous night were not able to manage the unexpected load of patients efficiently due to unavailability of nurses and other doctors. This was due to changeover of shifts and handing over those patients from the previous night who were still waiting in the unit. One doctor was allocated for early morning unexpected heavy loads of patients. He or she could call the stand-by team if the number of patients exceeded his or her capacity.

Other identified busy times were 13:00 to 19:00. It was observed that all the doctors were working from 08:00 to 16:00. Only two on-call doctors were working after 16:00 to 08:00 the next morning. It was decided that an eight-hour shift system would be used for the doctors working in the ED. Thus, doctors started their shift at 10:00 and 12:00. Their shift ended at 18:00 and 20:00 respectively, ensuring extra doctors in the afternoons, in addition to the two doctors on call. It was also observed that there was crowding of patients during lunchtime. In order to ensure enough coverage during peak times, the doctors working in the unit agreed to stagger their lunch times.

\section{f. Reorganising the academic programme}

Academic EDs are traditionally associated with inefficiency and long waits. ${ }^{9}$ The reason might be prolonged examination and more investigations in order to achieve theoretical standards. This was true for TDH as well. The majority of doctors working in this hospital and the medical students rotating in the ED had previously been working at urban tertiary hospitals. They had been ordering and waiting for unnecessary investigations and had discussions about interesting patient scenarios at any time. Academic meetings were organised at specific times in order to minimise the delays in patient care at peak times. The role of important investigations in different patient scenarios and the consequences of unnecessary laboratory tests were discussed in such meetings.

g. Notice of waiting time to patients

Literature shows the significance of notification and repeated information to the patients regarding delays. . $^{46}$ Notification of waiting time reduces patient dissatisfaction. ${ }^{14}$ It was decided that 
a notice would be placed at Reception to inform patients of the waiting time. The sister at Reception was also advised to inform patients verbally on arrival.

\section{h. Minor procedures}

It was postulated that if minor procedures, such as intravenous cannulation and minor suturing, were performed by trained nurses, doctors would sooner be able to attend to the next patient. ${ }^{11,14}$ International literature reports that a significant proportion of patients attending the ED need minor services that can be provided by trained nurses, thus decreasing waiting time by reduction of load on the doctor. ${ }^{4,17}$ If minor procedures are performed by trained nurses it can remarkably reduce ED waiting times. ${ }^{18,19}$ The nursing staff in the unit were happy to take on these tasks.

\section{i. Speaker phone}

It was realised that there was a delay in finding patients or their relatives for collateral history as well as the medical staff in emergencies. A speaker phone was installed to reduce these delays.

\section{j. Laboratory services}

Delays in both the collection of specimens and the availability of results were observed. Meetings were arranged with the laboratory personnel to request two-hourly specimen deliveries to the laboratory for urgent processing.

\section{k. Registration}

Time wastage at Registration was identified as one of the reasons for prolonged waiting time. ${ }^{20}$ It was observed that at certain times there was no clerk at Reception for registration. A meeting was held with the clerk supervisor to request that the Reception desk be manned by at least one person at all times (including tea and lunch breaks).

\section{Results}

As a result of the $\mathrm{Ql}$ cycles, the following aspects of the plan were carried out successfully: functional triage system, improvements in the process of up- and down-referrals, easy availability of stock, reorganisation of the duty roster and academic programme, announcement on waiting time to patients, nurses carrying out minor procedures, availability of a speaker phone and standardised management textbooks. However, goals regarding improvements in laboratory services and registration were not achieved in the present Ql cycles.

A total of 150 waiting times were evaluated. There were 50 waiting times each for May, September and December 2006. The total number of patients seen during the months of May, September and December 2006 was 3287,4066 and 3879 respectively. The average number of patients seen per 24-hour shift was May: 106, September: 136 and December: 125. In Figure 2 the frequencies of the waiting times for stable patients are shown for each month.

In May the longest waiting time was 1260 minutes, in September 480 minutes and in December 230 minutes. During May there were 37 stable patients and 13 unstable patients. The median waiting time for stable patients was 545 minutes (inter-quartile-range (IQR) 475-770). For unstable patients the median was 0 (IQR 0-5). During September
Figure 2: Waiting times for stable patients

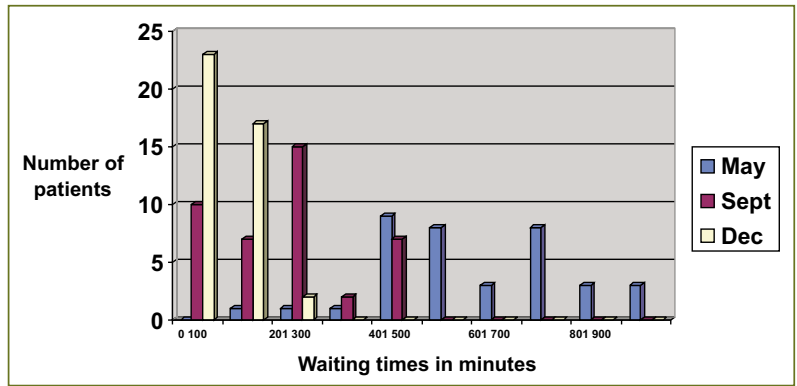

there were 41 stable and 9 unstable patients. The medians for the waiting times were the following: 230 minutes (IQR 105-290) for stable patients and 0 minutes (IQR 0-2) for unstable patients. For December the 50 waiting times collected were for 42 stable patients (median 89 minutes with IQR 60-125) and for 8 unstable patients (median 0.5 minutes with IQR $0-1.5$ ). The waiting times are summarised in Table I. The medians for stable patients are shown in Figure 3.

Figure 3: Median waiting times for stable patients

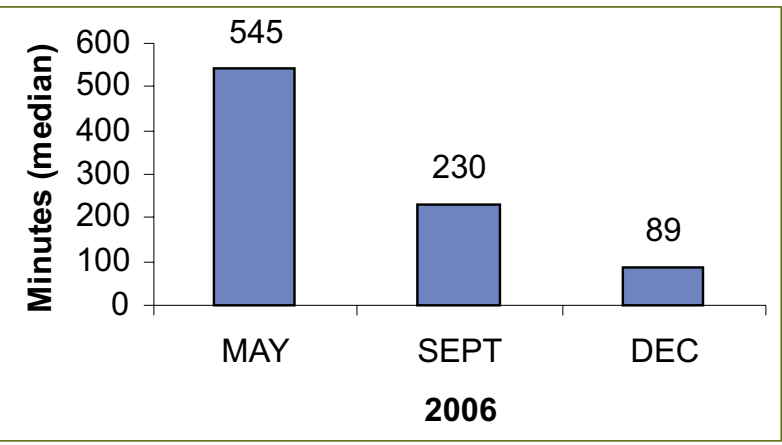

Table l: Waiting times

\begin{tabular}{|l|c|c|c|}
\hline Median* (stable patients) & May & September & December \\
\hline Minimum* (stable patients) & 200 & 230 & 89 \\
\hline Maximum* (stable patients) & 1260 & 480 & 230 \\
\hline Median* (unstable patients) & 0 & 0 & 0.5 \\
\hline Minimum * (unstable patients) & 0 & 0 & 0 \\
\hline Maximum * (unstable patients) & 30 & 3 & 2 \\
\hline
\end{tabular}

* Waiting time (minutes)

Statistical analysis using the Kruskall-Wallis $\mathrm{H}$ test showed a significant difference among the waiting times for stable patients for May, September and December $2006(p<0.001)$. There was no difference among the waiting times for unstable patients for May, September and December $2006(p=0.90)$.

\section{Discussion}

This paper documents how QI cycles were associated with a decrease in the waiting times for stable patients at TDH ED, using the available resources. The study helped to identify and rectify some problems. This study was a source of encouragement and motivation for the QI team. The QI cycle works best if the team is appropriate and the team members understand the process and cooperate with each other. ${ }^{11}$ The biggest factor in the reduction of waiting time and improved 
performance was a number of measures rather than one specific measurement. ${ }^{21}$ This improvement was achieved by an effective team effort in this Ql exercise.

The QI team identified the need for an effective triage system. The process of sorting patients according to their severity of illness is called triage. ${ }^{8}$ Literature shows the importance of effective triage in reducing waiting times and improving patient satisfaction. ${ }^{14}$ The need for effective triage to recognise unstable patients for immediate attention was identified. Before this study, unstable patients were identified according to ATLS criteria. However, the triage system was not functioning well before the interventions, as is evident from Table I that depicts a wait of 30 minutes in May for an unstable patient. This system was improved by training and monitoring. A trained professional nurse for triage was identified for each shift and could call the MP, if required. The longest waiting times for unstable patients improved to three and two minutes in September and December 2006 respectively. However, there was no significant change in the median waiting times of unstable patients.

The ED is the gateway that a majority of patients use to access care within hospitals. ${ }^{10} \mathrm{ED}$ crowding and delays are a universal problem. ${ }^{2}$ Prolonged waiting times are associated with reduced patient satisfaction, increased risk of leaving the hospital without being seen and poorer long-term health outcomes. ${ }^{9,15}$ However, repeated notification of delays and length of wait improves patient satisfaction. ${ }^{4}$ Verbal information given by the sister and the written notice placed at Reception were useful. This information was also important as the patients could plan and utilise their time for some other activities instead of just sitting and waiting. ${ }^{6}$ The staff welcomed this change and informed the researcher that they had to deal with relatively fewer complaints regarding waiting times. However, this may not be the result of one intervention but the effect of overall reduction in waiting times.

MPs working in TDH ED unit were positive about the suggested interventions in the referral system, availability of standardised management textbooks and reorganising the duty roster and academic programme. All those changes were implemented after motivational talks. International studies show the reduction in waiting time by such measures. ${ }^{4,17}$

Interventions regarding availability of stock and minor procedures to be performed by trained nurses were successful. Nurses in the unit carried out those tasks successfully. Those changes were implemented after obtaining literature evidence of reduction in waiting time by similar measures. ${ }^{11,14,16}$

International literature shows various waiting times in EDs. In Chicago the mean waiting time was 38 minutes. ${ }^{4}$ According to California EDs their average waiting time was 56 minutes. ${ }^{5}$ The above-mentioned studies show shorter waiting times than those in the results of the South African study. In the Queen Elizabeth Hospital ED in Barbados (West Indies), the median waiting time to be seen by a doctor was 178 minutes, compared to the 89 minutes median time at TDH ED in December 2006. ${ }^{6}$ Presently, South African targets for stable patients are up to 120 minutes. ${ }^{8}$ In the QI study carried out in TDH ED, this goal was achieved by December 2006.

Most aspects of the plans were carried out successfully. However, goals regarding improvement in laboratory services and registration were not achieved. This might be due to not having any representatives from those departments in the QI team.

\section{Bias and limitations}

This was a small study with limited data. A truly representative sample would have been obtained by randomly sampling days of the week taken over the entire period. However, this was a prospective study with limited resources and no funding; therefore, a huge sample collection was not possible.

The sample was skewed; therefore, the Kruskall Wallis $\mathrm{H}$ test was performed showing median value.

The performance can improve if workers know about the audit. To minimise this temporary enhancement the data collection was kept secret and only one MP was used. There were no benefits or rewards for a successful QI cycle to prevent personal bias in data collection.

The number of nursing staff and MPs working in the ED did not change during the study period. However, the skills of the staff would have been improved with the passage of time, due to more exposure, experience and knowledge in the ED system.

Waiting times can be affected if the turnout of patients varies significantly. To overcome this confounding effect all data collection were done at the beginning of the month concerned when the ED was relatively busy.

\section{Suggestions}

Ql is teamwork. The team should be selected carefully. Motivated personnel representing all relevant departments can make this exercise more successful.

Each $\mathrm{Q}$ cycle is unique. QI cycles can be useful in any ED, but strategies would vary according to the circumstances.

\section{Conclusion}

The QI cycles identified some problems causing prolonged waiting times in TDH ED. The following aspects of the plan were carried out successfully: functional triage system, improved referral system, availability of reference books and speaker phone, easy availability of stock, reorganising the duty roster and academic programme, notification of waiting time and nurses carrying out minor procedures. However, goals regarding registration and laboratory delays could not be successfully achieved.

This study shows the significance of QI cycles in improving waiting times of stable patients at TDH ED without any additional financial or human resources.

\section{Acknowledgements}

The authors acknowledge the kind support, guidance, information and advice provided by Dr Sarie Oosthuizen and Prof David Cameron during this study. Our thanks are due to Dr Selma Smith and Dr Patrick Kenny for fruitful comments on this article.

\section{References}

1. Gottschalk SB, Wood D, DeVries S, Wallis LA, Bruijns S. The Cape triage score: A new triage system in South Africa. Proposal from the Cape triage group. Emerg Med J 2006;23:149-53.

2. Shute N, Marcus M. Crisis in the ER. US News and World Report. 2001;10:54-61.

3. Fottler MD. Managing patient waits in hospital emergency departments. Health Care Manag 2002;21(1):46-61.

4. Thompson DA, Yarnold PR, Williams DR, Adams SL. Effects of actual waiting 
time, perceived waiting time, information delivery and expressive quality on patien satisfaction in the emergency department. Ann Emerg Med 1996 Dec;28(6):657-65.

5. Lambe $S$, Washington DL, Fink A, et al. Waiting times in California's emergency departments. Ann Emerg Med 2003 Jan;41(1):35-44.

6. Banerjea $\mathrm{K}, \mathrm{Carter} \mathrm{AO}$. Waiting and interaction times for patients in a developing country accident and emergency department. Emerg Med J 2006;23:286-90.

7. Jones $\mathrm{G}$. Ways for reducing the waiting times for patients in A\&E. Nurs Times 1996; 92(11):31-2.

8. Mac Farlane C, Naidoo S. Triage in South African hospitals. S Afr Med J 2006;96(7): 570-1.

9. Spaite DW, Bartholomeaux F, Guisto J, et al. Rapid process redesign in a universitybased emergency department: Decreasing waiting time intervals and improving patient satisfaction. Ann Emerg Med 2002 Feb;39(2):168-77.

10. Waseem M, Ravi L, Radeos M, Ganti S. Parental perception of waiting time and its influence on parental satisfaction in an urban pediatric emergency department: Are parents accurate in determining waiting time? South Med J 2003;96(9):880-3.

11. Schwarz M, Suzanne E, Rowe JE. A team approach to quality improvement. Fam Pract Manag 1999;6(4):25-30.

12. Mash B, editor. Handbook of family medicine. SA: Oxford University Press;2000.
13. American College of Surgeons; Committee on Trauma. Advanced trauma life support for doctors. Chicago (USA);1997.

14. Choi YF, Wong TW, Lau CC. Triage rapid initial assessment by doctor (TRIAD) improves waiting time and processing time of the emergency department. Emerg Med J 2006;23:262-5.

15. Goodacre S, Webster A. Who waits longest in the emergency department and who leaves without being seen? Emerg Med J 2005;22:93-6.

16. Naumann S, Miles JA. Managing waiting patients' perception; the role of process control. J Manag Med 2001;15(5):376-86.

17. Grouse A, Bishop R. Non-medical technicians reduce emergency department waiting times. Emerg Med 2001;13:66-9.

18. Cooke MW, Wilson S, Pearson S. The effect of a separate stream for minor injuries on accident and emergency department waiting times. Emerg Med J 2002;19:28-30.

19. Byrne G, Richardson M, Brunsdon J, Patel A. An evaluation of the care of the patients with minor injuries in emergency settings. Accid Emerg Nurs 2000;8:101-9.

20. Travers JP, Lee FCY. Avoiding prolonged waiting time during busy periods in the emergency department: Is there a role of senior emergency physician in triage? Eur $\mathrm{J}$ Emerg Med 2006; 13(6):376-86.

21. Munro J, Mason S, Nicholl J. Effectiveness of measures to reduce emergency department waiting times: A natural experiment. Emerg Med J 2006;35-9. 